\title{
Acoustic Field Distortions Caused by Helium-Filled Balloons
}

\author{
L. Majkut*, R. Olszewski And M. Pluta \\ AGH University of Science and Technology, Department of Mechanics and Vibroacoustics \\ Al. A. Mickiewicza 30, 30-059 Krakow, Poland
}

\begin{abstract}
In this paper, an automated measurement system was proposed that uses a probe hung below a remotely controlled balloon to measure parameters of the acoustic field in the whole volume of a room. The study was conducted to estimate distortions that such system may cause in the acoustic field at the measurement point. The most significant source of the distortions will be the buoyancy element - one large balloon or a number of smaller ones. This paper considers different spatial configurations of spherical balloons with regards to alterations they cause in the acoustic pressure levels measured below them. Interior of a room without and with a group of spherical balloons was modeled using the boundary element method. Using a larger number of smaller spheres instead of a single large one did not significantly reduce the related distortions. However, using two spheres slightly decreased distortions when the distance between the balloons and the recording point was relatively small.
\end{abstract}

DOI: 10.12693/APhysPolA.125.A-84

PACS: 43.55.Br, 43.55 Hy, 43.55.Nd, 43.55.Mc

\section{Introduction}

An automated system for measurements of acoustic field in the whole volume of a room was proposed in [1,2]. The purpose of the system is to be capable of measuring selected parameters in a free three-dimensional mesh of points chosen in the whole volume of the room. A measurement probe (i.e. microphone) is hung below remotely controlled balloons (one or more).

Due to relatively small size of a microphone in relation to the wavelength, it is always assumed that in a range of sound wave lengths, presence of microphone has a negligible influence on the acoustic field and the value of acoustic pressure in measurement points. The same cannot be said when the microphone is moved using an instrumentation, e.g. in a form of an arm, a blimp, or a group of balloons [1,2]. Knowledge about the range of influence of measurement instrumentation elements on acoustic field produced by sound wave sources is practically limited to the free field. There is a large contribution to this knowledge from Polish researchers [3] whose works illustrate phenomena occurring in acoustic field under the influence of elements that form an obstacle on a path of sound wave propagation.

In case of acoustic field inside a room, it is difficult to point to any works that would directly describe an impact of measurement instrumentation on the observed acoustic field. A prior research in this area resulted in application of a manipulator in an anechoic chamber for the purpose of automation of acoustic measurements [4]. It should be noted, though, that in the case of an anechoic chamber, the acoustic field is the free field, without reflected waves. Experience and knowledge obtained in

*corresponding author; e-mail: majkut@agh.edu.pl such a case cannot be directly applied to rooms where reflected waves are present.

As a part of the design phase of the automated measurement system for room acoustics, a study was conducted to estimate distortions that such a system may cause in the acoustic field. The most significant source of distortions will be a large buoyancy element that will allow the probe to be moved to any location inside the room. Such an element can occur in various forms. This paper considers different spatial configurations of spherical buoyancy elements (i.e. helium-filled balloons) with regards to alterations they cause in the acoustic pressure levels measured below them (by microphone hung from balloons).

\section{Automated measurement system for room acoustics}

Acoustic properties of a room greatly influence its usability. If they turn out to be unsatisfactory, a highly expensive and labor-intensive adjustments may be necessary to correct any design or adaptation flaws $[5,6]$. The corrections are based on acoustic measurements of the existing room as well as on the results obtained from the computational model of the room. Measurement is necessary for computational model alignment. Based on numerical model with different adjustments, the appropriate adaptation of the corrected room is proposed. Therefore, it is vital that those two groups of methods produce consistent results when applied to the same room.

The typical measurement procedure is described in $[5,6]$. Measurements were conducted in a church with no people present. The measurement procedure involved manual placement of a microphone on a stand in selected points and can provide information regarding only a small part of the acoustic field in the whole space of the church. In this particular case, the microphone was placed in 13 different locations. Along with a microphone, an omnidirectional sound source, an audio inter- 
face, and a dedicated software were used. The impulse response was recorded and a number of room parameters typically used to evaluate acoustic quality of rooms were calculated. More measurement locations would lead to more realistic model of the room and more accurate predictions about suitable adaptation [7,8] (or at least computational model alignment).

The proposed measurement system for room acoustics (Fig. 1) would provide automation of both the measurement cycle in a single location and moving the probe to different location. Two subsystems need to work together for this purpose: stationary and mobile. The stationary one will include an omnidirectional sound source, a power amplifier, an audio interface, a computer, a stationary part of the positioning system, a data transfer system, and an autonomic power supply unit. The mobile one will consist of one or more remotely controlled balloons carrying a measurement unit, a mobile part of the positioning system, a data transfer system, and batteries. The system will allow to efficiently conduct detailed measurements in various rooms, so that simulation results used in room acoustics could be verified and possibly improved (by model alignment).

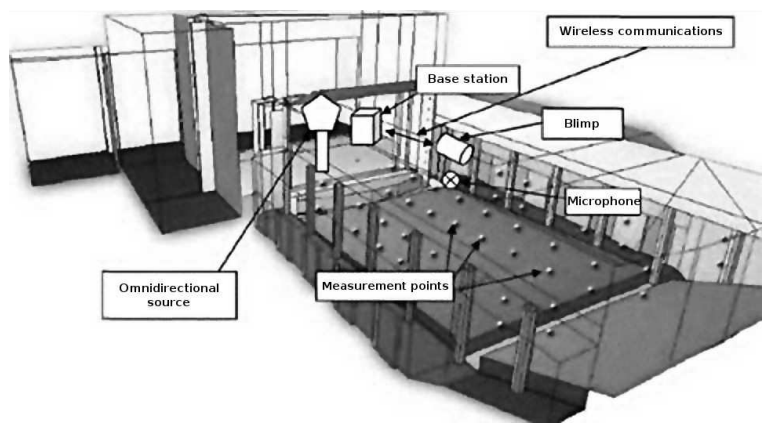

Fig. 1. An automated measurement system for room acoustics.

\section{Spatial configuration of balloons}

The source of buoyancy in the automated measurement system - a hypothetical device for transporting the measurement microphone throughout an actual room - will be a number of helium-filled mylar shell balloons. There are practical constraints for such a source, two of which are the most important:

1. it must be able to lift a sufficient mass of equipment $(\sim 0.5 \mathrm{~kg})$;

2. its linear dimensions should not significantly exceed $1 \mathrm{~m}$, in order to enable maneuvering in enclosed spaces.

On the basis of the first condition, one can obtain the following relationship between a number and size of balloons, assuming equal size of all balloons, mylar shell thickness of $1.8 \times 10^{-5} \mathrm{~m}$, mylar density of $1.39 \times 10^{3} \mathrm{~kg}$ $\mathrm{m}^{-3}$, helium and air temperature of $293 \mathrm{~K}$, and helium and air pressure of $1.01 \times 10^{5} \mathrm{~Pa}$ :

$$
\left(\frac{\Delta \rho}{3}\right) r^{3}-d \rho_{\mathrm{m}} r^{2}-\frac{m_{\mathrm{L}}}{4 \pi N}=0,
$$

where $r$ is the single balloon's radius, $N$ is the number of balloons, $\Delta \rho$ is air and helium density difference, $d$ is the mylar shell thickness, $\rho_{\mathrm{m}}$ is the mylar density, and $m_{\mathrm{L}}$ is the mass of load (equipment).

From Eq. (1) the number of balloons and their corresponding radius can be determined. The relationship between the number of balloons and their radius is shown in Fig. 2.

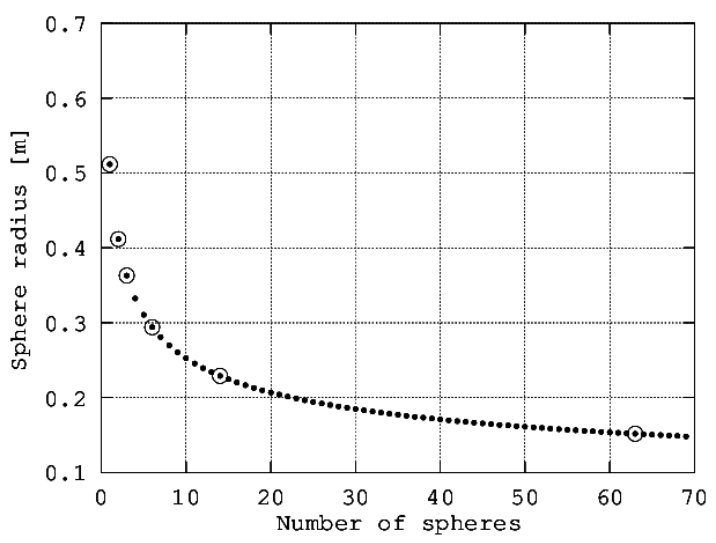

Fig. 2. Relation between number and size (radius) of spherical balloons with mylar shell able to lift a load of $0.5 \mathrm{~kg}$.

TABLE

Spatial configurations of balloons selected for the study ( $r$ - balloon radius, $N$ - number of balloons).

\begin{tabular}{c|c}
\hline \hline & $r=0.512 \mathrm{~m}, N=1$ \\
\hline 0 & $r=0.412 \mathrm{~m}, N=2$ \\
\hline 80 & $r=0.363 \mathrm{~m}, N=3$ \\
\hline 898 & $r=0.294 \mathrm{~m}, N=6$ \\
\hline 898 & $r=0.152 \mathrm{~m}, N=63$ \\
\hline
\end{tabular}

The second condition can be met by arranging spherical balloons according to the face-centered cubic (fcc) crystal system lattice, which is the most dense sphere arrangement with approximately $74 \%$ of the total volume being occupied by the spheres, as can be calculated from the formula:

$$
\frac{V_{\mathrm{S}}}{V_{\mathrm{C}}}=\frac{N \frac{4}{3} \pi R^{3}}{a^{3}},
$$

where $V_{\mathrm{S}}$ is the volume of all spheres in a unity cell, $V_{\mathrm{C}}$ is the total volume of a unity cell (cubical in this case), $N$ is the number of spheres in a unity cell ( $N=4$ in fcc), 
$R$ is the radius of a sphere, and $a$ is the side of a cube $(a \sqrt{2}=4 R)$.

In order to reduce linear dimensions of a group of balloons, the group should be formed into a cube.

Simulations were performed for 6 different configurations: 1, 2, 3, 6, 14, and 63 balloons, denoted by circles in Fig. 2 and shown in Table.

\section{Simulation study}

Interior of a room with a group of spherical balloons causing a distortion in an acoustic field was modeled using a boundary element method (BEM). BEM is a numerical method that allows to find an approximate solution of the Helmholtz equation $[9,10]$. The model assumes that medium in which an acoustic wave propagates has the following properties: continuity and homogeneity, perfect fluidity, perfect elasticity, lack of internal thermal inertia, and lack of viscosity. Computations are conducted in the frequency domain. The results are amplitude and value of phase shift angle of the acoustic wave. It is possible to consider diffraction and interference of multiple direct and reflected waves. However, in this study only interference is considered. The diffraction that occurs on the boundary between the air outside and the helium inside the balloon was omitted.

In calculations, the following parameters of the room were adopted: $l=12 \mathrm{~m}, w=9 \mathrm{~m}, h=7 \mathrm{~m}$ (Fig. 3 ). 7 simulations were performed for: an empty room - as a reference data, and 6 different groups of spheres described in Section 3 (Table). It was assumed that for a given medium (air), the density amounts to $\rho=1.221 \mathrm{~kg} \mathrm{~m}^{-3}$ and the sound propagation velocity $c=343 \mathrm{~m} \mathrm{~s}^{-1}$. For the sake of simplicity, it was assumed that the acoustic impedance of all walls is the same and amounts to $Z_{\mathrm{S}}=$ $4750-520 \mathrm{i}$, while the acoustic impedance of the balloon boundary equals $Z_{\mathrm{Z}}=1200-90 \mathrm{i}$.

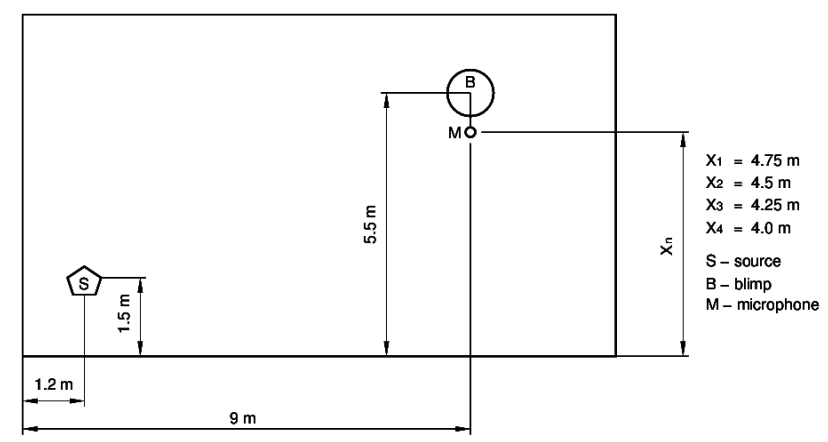

Fig. 3. Simulated room with a sound source location, observation locations, and distorting element (balloon) location.

A source of spherical wave with acoustic power of $L_{N}=75 \mathrm{~dB}$ was placed in the point with the following coordinates: $x_{g}=1.2 \mathrm{~m}, y_{g}=1.0 \mathrm{~m}, z_{g}=1.5 \mathrm{~m}$. In all 6 cases, the geometric center of the group of spheres was located in the point with the same coordinates: $x_{e}=9.0 \mathrm{~m}$, $y_{e}=6.0 \mathrm{~m}, z_{e}=5.5 \mathrm{~m}$. Four observation (recording) points were located $0.75 \mathrm{~m}, 1.0 \mathrm{~m}, 1.25 \mathrm{~m}$, and $1.5 \mathrm{~m}$ below the center of the group of spheres, in the point for which $x_{e}=9.0 \mathrm{~m}, y_{e}=6.0 \mathrm{~m}, z_{e}=4.75 \mathrm{~m}, 4.5 \mathrm{~m}, 4.25 \mathrm{~m}$, and $4.0 \mathrm{~m}$, that is in close vicinity of the distorting element, but in such a manner that it was "visible" from the source and reached by the direct (non-reflected) wave. These four points were selected in order to study differences between possible placements of the microphone in the real measurement system. Calculations were performed in the frequency range from $20 \mathrm{~Hz}$ to $1 \mathrm{kHz}$ with an increase of $\Delta f=10 \mathrm{~Hz}$. Sound levels were calculated according to the following formula:

$$
L_{p}=20 \log _{10} \frac{p}{p_{0}},
$$

where $p$ is the value of the acoustic pressure in the measurement point and $p_{0}=2 \times 10^{-5} \mathrm{~Pa}$ is the value of the reference acoustic pressure

\section{Numerical results}

On the basis of the simulation results, two parameters have been calculated for each observation point and each spatial configuration: the maximum acoustic pressure level change

$$
D_{\mathrm{Max}}=\max \left|L_{D i}-L_{E i}\right|
$$

and the generalized acoustic pressure level change

$$
\begin{gathered}
D_{\mathrm{Gen}}=10 \log _{10}\left(\sum_{i=1}^{n} 10^{0.1 L_{D i}}\right) \\
-10 \log _{10}\left(\sum_{i=1}^{n} 10^{0.1 L_{E i}}\right),
\end{gathered}
$$

where $L_{D i}$ is the acoustic pressure level in distorted field for $i$-th analyzed frequency of excitation $L_{E i}$ is the reference acoustic pressure level (without balloons) for $i$-th frequency, and $n$ is the number of frequencies in the analyzed range $(n=99)$. $D_{\text {Max }}$ represents the change in acoustic pressure level at a single excitation frequency the one at which the change is the largest. $D_{\text {Gen }}$ represents the change averaged for all the frequencies (20-1000 $\mathrm{Hz}$ ). The obtained values are presented in Figs. 4 and 5 .

Results vary between numbers of balloons and observation points. Generally, the lowest changes in the acoustic pressure level are observed $1 \mathrm{~m}$ below the geometric center of the group of spheres, except for 3 spheres where changes are getting lower with increasing distance from the spheres; this configuration seems to be the best one to use if an equipment is going to be suspended far from the balloons. Using larger number of smaller spheres does not significantly reduce distortions, probably because of larger area of the reflecting surface. However, using 2 spheres slightly decreases the changes for the smallest distance from the center of the group of balloons $(0.75 \mathrm{~m})$. The largest changes in the acoustic pressure level (above $40 \mathrm{~dB}$, as shown in Fig. 4) are observed in the following cases:

- 6 spheres and distance $0.75 \mathrm{~m}$, excitation frequency $660 \mathrm{~Hz}(45.7 \mathrm{~dB})$, 


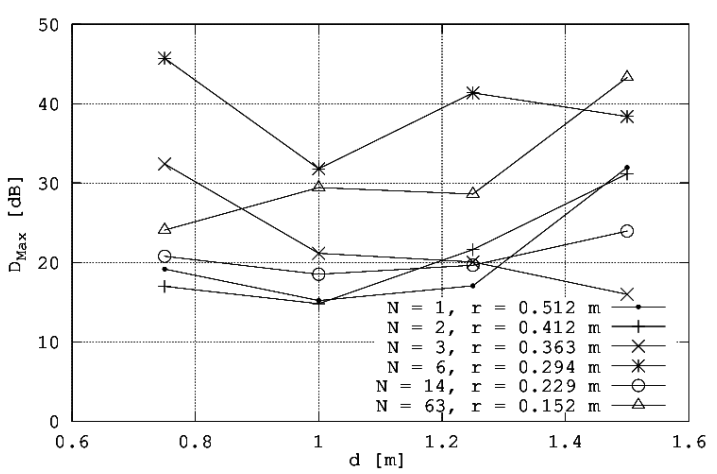

Fig. 4. The maximum acoustic pressure level change in individual observation points $(d-$ distance between the center of the group of balloons and the microphone) for different configurations of spheres.

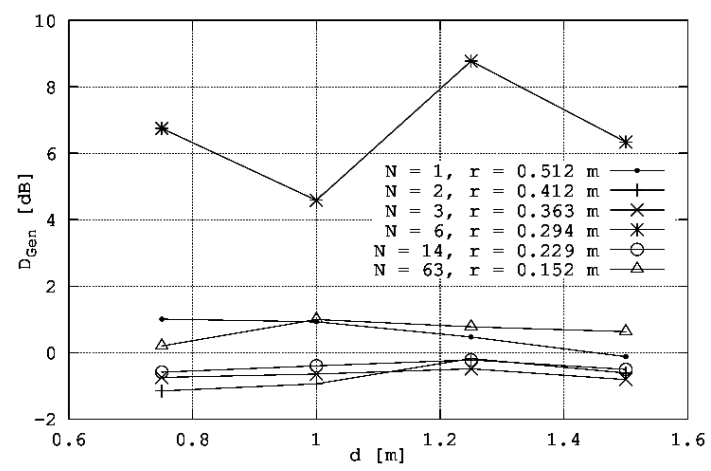

Fig. 5. The generalized acoustic pressure level change in individual observation points $(d-$ distance between the center of the group of balloons and the microphone) for different configurations of spheres.

- 63 spheres and distance $1.5 \mathrm{~m}$, excitation frequency $790 \mathrm{~Hz}(43.3 \mathrm{~dB})$,

- 6 spheres and distance $1.25 \mathrm{~m}$, excitation frequency $660 \mathrm{~Hz}(41.4 \mathrm{~dB})$.

\section{Conclusions}

The obtained results illustrate changes in acoustic field resulting from introduction of spherical balloons in a modeled room. It can be stated that the element which is not a part of the room "equipment", but is introduced inside, affects values measured in the acoustic field depending on its shape, dimensions, and coordinates of location. Using larger number of smaller balloons instead of a single bigger one, does not significantly reduce, and can even increase distortions. However, using two balloons slightly decreases distortions. The second analyzed effect was the influence of microphone location below the center of the group of balloons on measurement accuracy. In the best case with respect to the number of balloons (i.e. 2 balloons), the minimal errors were observed for the distance equal to $0.75 \mathrm{~m}$.

The next step will be to study the relation between the distortions and the overall shape of the group of balloons, as this seems to have more impact on the acoustic field than the radius of a single sphere. In order to obtain more complete understanding of the phenomena and their influence on the measured values in the acoustic field, it is necessary to analyze acoustic field distribution in many observation points, with different geometrical configurations of the group of balloons taken into account. The best design, chosen in the optimization procedure that minimizes the distortions, should be studied experimentally. Final results will allow to estimate the impact of distortions caused by measurement instrumentation on the observations and point out ways to minimize this impact by assuming proper design solutions.

\section{References}

[1] B. Borkowski, R. Olszewski, M. Pluta, Studia i Materiaty Polskiego Stowarzyszenia Zarzadzania Wiedza 45, 33 (2011).

[2] B. Borkowski, R. Olszewski, M. Pluta, Logistics 6, 299 (2011).

[3] S. Weyna, Archiv. Acoust. 28, 191 (2003).

[4] T. Kamisiński, A. Flach, Archiv. Acoust. 31, 235 (2006).

[5] T. Kamisiński, K. Kosała, Tech. Trans. Archit. 108, 2-A/1 115 (2011).

[6] K. Kosała, Archiv. Acoust. 37, 23 (2012).

[7] I. Witew, P. Dietrich, D. de Vries, M. Vorlander, ISRA 2010, Melbourne 2010.

[8] I. Witew, M. Vorlander, Proc. Inst. Acoust. 33, 23 (2011).

[9] R. D. Ciskowski, C. A. Brebbia, Boundary element methods in acoustics, Elsevier, London 1991.

[10] S. Kirkup, The Boundary Element Method in Acoustics. 